\title{
Comparing a Novel Antenatal Program with a Standard in Disadvantaged Women at Psychosocial Risk: A Randomized Controlled Study
}

\section{Ortíz Collado Maria Assumpta}

Haute école de santé La Source Laussane, Vaud Switzerland

*Corresponding author: Ortíz Collado Maria Assumpta, Ph.D, Haute école de santé La Source, Laussane, Vaud Switzerland, Tel: 0216413836 ; Fax: +41 216413838; E-mail: m.ortizcollado@ecolelasource.ch

Received date: March 28, 2014, Accepted date: October 28, 2014, Publication date: Novemver 07, 2014

Copyright: (c) 2014 Assumpta OCM. This is an open-access article distributed under the terms of the Creative Commons Attribution License, which permits unrestricted use, distribution, and reproduction in any medium, provided the original author and source are credited.

\begin{abstract}
Purpose: To evaluate participant's satisfaction with two models of antenatal childbirth preparation program and effects in women at psychosocial risk. The risk variables included depressive symptoms, stressful events, lack of social support and, low economic level.

Design, setting and participants: A blinded randomized controlled and multi-centre trial comparing a novel program intervention to standard program. Women found to be at psychosocial risk were distributed in two groups: an experimental group (EG) or a control group (CG) and evaluated in two times measurement: 1) during pregnancy (<20 weeks) before the routine ultrasound visit and, 2) four weeks after delivery by parcel post. The sample was 184 pregnant women and their partners.
\end{abstract}

Intervention: 10 group sessions in experimental group, focusing on body awareness sensations with humanistic mind-body approach (like mindfulness) in direction to constructing of an individualized childbirth model. Sessions lasted two hours and 15 minutes each during pregnancy, with one individual telephone conversation between sessions. In the control group intervention, the participants were free to choose the standard model of antenatal education: 10 two-hour sessions focused on childbirth preparation and breast feeding, including obstetrical prophylaxis and relaxation.

Results: Satisfaction was higher in the experimental group for questions about understanding symptoms of pregnancy: 4 in the $C G$ and 38 in the $E G$ ( $p=0.05$ Pearson Chi-Square and $p=0.05$ Fisher's Test); understanding all information received: 3 in the $C G$ and 37 in the $E G(p=0.02$ Pearson Chi-Square and $p=0.02$ Fisher's Test); expressing feelings: 0 in the CG and 18 in the $E G$ ( $p=0.04$ Pearson Chi Square and $p=0.03$ Fisher's Test); and communicating with the baby: 3 in the CG and 38 in the $E G(p=0.05$ in Pearson Chi-Square and $p=0.05$ Fisher's Test). The difference in preterm births between groups is significant $(P=0.003)$ with only three premature baby in the experimental group and thirteen in the control group. The weight of the babies was lower in the control group compared to the experimental group $(p=0.01)$.

Conclusions: A novel antenatal program that included a psychosomatic approach about all pregnancy sensations (mother and baby) produces higher satisfaction in participants. Program can help to preventing the risk of prematurity in women at psychosocial risk but needs to be studied with a larger sample. Early detection realized on the occasion of the routine ultrasound visit, before 20 weeks pregnancy was an advantage, and suggests effectiveness in the selection of cases at risk.

Keywords: Pregnant women; Antenatal program; Psychosocial risk; Childbirth

\section{Introduction}

Pregnant women often prepare for the birth of their babies and normally go to health care centers to receive the standard care. However, some women think that there is no reason to participate in the prenatal courses; some even forgets parts of the care protocol. A few authors have found insufficient medical monitoring and low participation in any sessions on maternity preparedness in pregnant women at psychosocial risk [1,2]. However, compared with the general population, women with psychosocial risk tend to have more complications such as premature and underweight babies [3], higher prevalence of postpartum depression [2], also often have additional stress caused by an ailing economy, unemployment, separation or divorce [4].

In the present study, the psychosocial risk in pregnancy variable is issued from the assessment of the following factors: a) depressive symptoms, b) stressful events, c) low economic level, and d) lack of social support. The aforementioned variables have already been associated with childbirth outcomes, but were rarely included together in the research on evaluation of an antenatal program.

Care for mothers at psychosocial risk is very important and their participation in antenatal programs can be a way to encourage to adherence to the mother-baby care protocol. For example, some authors observed a relation between the use of antenatal services and 
care and birth outcomes [5]. While a Spanish group study expected that $40 \%$ of pregnant women would not join any antenatal courses, it was concluded that only $7 \%$ of them rejected it, and moreover, the attendance to the sessions was more than $50 \%$. However, the type of services offered in the health centers, its reputation and accessibility, and the cultural level of participants may influence the decision to perform an antenatal course [6]. It is likely that parents who are at psychosocial risk require programs tailored to their needs.

On the other hand, some authors suggest that the parental transition in men needs to be more contemplated in antenatal course; they represent the only support in many cases and were not always heard [7]. Perhaps, it is worth assessing program participation together with the adherence to antenatal care protocol for pregnant women, as there are problems that cannot be solved in antenatal courses. It is important to consider that women who do not make antenatal visits according to protocol may have undetected infections with risk for themselves and the baby [8]. A childbirth preparation program aimed at couples of low socioeconomic status could help to detect psychosocial problems rarely identified during medical visits. Many pregnant women with psychosocial risk suffer from anxiety and depression with implications for their babies: high fetal activity, low birth weight and prematurity [9-11]. These problems could be easily detected from the comments and questions that women often express during antenatal meetings led by the nurse midwifes. However, antenatal programs should offer a design and intention to inspire the participation of couples. They need to upgrade preparedness interventions at birth, because their main objective (to decrease labor pain) has lost justification, the population needs have changed, and the current problems are different [6]. Training to reduce pain in childbirth is still favorable; however, other problems also become very important, for example, reducing stress levels, increase social support, or reduce depressive symptoms.

The authors who have evaluated antenatal programs, which main objective is the mental health of women, have achieved significant results in variables such as the adaptation to birth and mothers' selfesteem [12], reducing anxiety [13] and reducing depressive symptoms $[14,15]$. In a randomized study on the impact of a program based on weekly antenatal massage, a lower prevalence of premature births and maternal depression was observed [16]. If the mother is protected from depression, her pregnancy will carry less risk, as circumstances of physical and mental stress favor preterm delivery and lower weight of babies at birth $[17,18]$. Indeed, low birth weight and prenatal depression are associated variables [10].

Most studies on antenatal courses aimed at improving the wellbeing and mental health of pregnant women are oriented on theories such as cognitive-behavioral theory [19-22], interpersonal theory $[14,23,24]$, or follow a psycho-educational model [15,25-27]. In some of those studies, antenatal programs were organized with two different professionals: a) the nurse-midwife, who takes care of the physiological pregnancy, and b) a psychologist or a social worker, who deals with the emotional aspect. To date, there are no known antenatal programs in which the intervention was inspired by an integrative model of awareness body sensations as that of Tourné [28]. Tourne's model enables a single nursing professional to intervene in all aspects. However, this means that the central objective of the program is not the pain or the physical condition of women, aspects already valued in standard antenatal classes. It is about going beyond this approach, focusing on the couple as future parents, enhancing the experience with the baby in utero and creating a supportive group environment.
The intervention sessions according to Tourne's model, give rise to the autonomy of the participants who have to find their own individual model in the experience of sensations and communication with the partner and the baby. Obviously this type of intervention is humanistic.

Pregnant women with depressive symptoms are more frequent distressing physical symptoms and lower self-esteem in a study that examine a multi-factorial model of body-related experiences during pregnancy; this study conclude that low self-esteem and low appreciation of their new body image are associated [29]. Other study with a program that includes body awareness with physical exercise in pregnancy observed influences in the perception of self-efficacy, and simultaneously a practice exercise improves the body's internal competence [30]. However, few investigations contemplated the benefits of antenatal exercise program incurred bodily consciousness. The conscious exercise adapted to motherhood is to balance the physical and mental attitude can contributes to emotional balance.

The organization and content of the sessions of the present study was carried out following the method of Tourné [28], that facilitates awareness of sensations and control of certain symptoms by a better understanding thereof. We hypothesize that according to this approach, the satisfaction of the participants can be greater than in the control group and other variables than preterm birth would diminish. Assistance at birth preparation sessions with the described attributes (the method of Tourné) should positively influence of the mother, father and the baby, as well as the adherence to the protocol which will encourage the involvement of professional care.

\section{Goals}

The main objective of this study is to evaluate participant's satisfaction with two models of antenatal childbirth preparation program and effects in women at psychosocial risk using a humanistic mind-body approach (Tourne's method). The evaluation focuses on three main variables: satisfaction intervention in women, premature birth and the weight of babies at birth. It also evaluates a secondary variable, which is the adherence to the clinical protocol.

\section{Materials and Methods}

\section{Study design}

This is a clinical controlled trial which will compared a novel antenatal education program based on a psychosomatic humanistic approach recently developed and standard program. The intervention aimed at preparing couples-where women are considered at psychosocial risk- to the childbirth/parenting event. The flow of the study design is outlined in Figure 1. The recruited participants are allocated to the intervention (experimental group-EG) or to the standard care group (control group-CG). Both groups are followed prospectively throughout pregnancy. The outcomes are compared between groups at their recruitment between 10-16 weeks of pregnancy (T1) and between 5 to 10 weeks postpartum (T2). The participants were selected by a statistician who never saw the women, using a computerized system based from the psychosocial risk identified in the first interview. The statistician was not connected to the rest of the study. 
Citation: Assumpta OCM (2014) Comparing a Novel Antenatal Program with a Standard in Disadvantaged Women at Psychosocial Risk: A

\section{Flow Diagram}

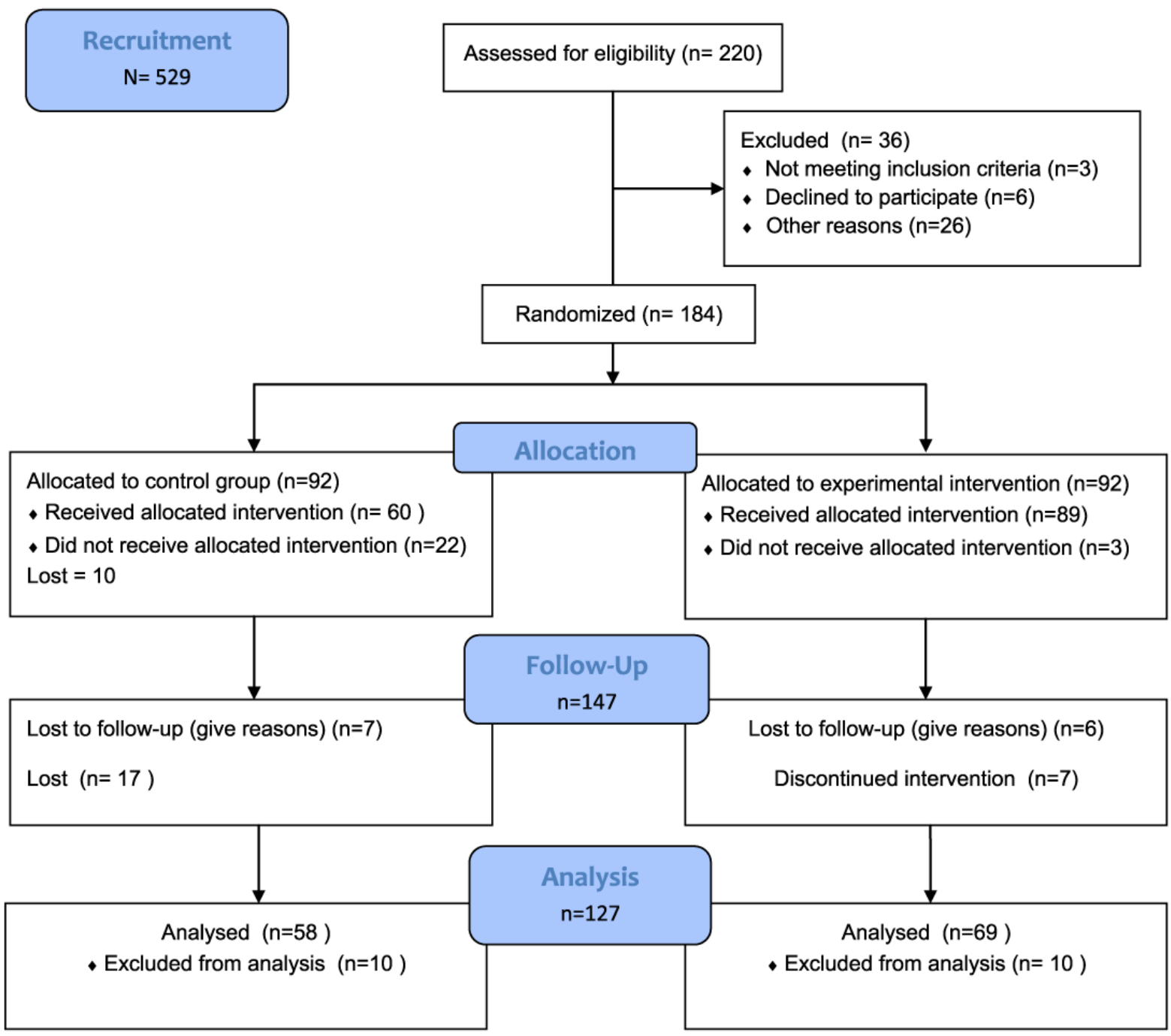

Figure 1: Flowchart.

\section{Recruitment}

During periods of recruitment, the researcher went to ultrasound waiting room, where she received a list of all pregnant women who did not surpass the $16^{\text {th }}$ week of pregnancy. The study was explained directly to each woman requesting her participation. The selection interview took part in an adjacent office, which was arranged for the study, and with their signed consent. At the end of the interview, they were given the first set of questionnaires that they would have to answer and return immediately. Each week the interviews identified by codes are sent to the statistician. Women with psychosocial risk were retained, the others not. Then they proceeded to the random distribution to participate in the control group or the experimental one, respecting the level of risk. A phone call served to inform people about the group participation.
All antenatal questionnaires were retained by the first statistician up to the end of the interventions of all groups; at the end of all interventions, he made a transfer of documents to a second statistician, who introduced the rest of the data. Finally, a third statistician worked on analyzing of all variables. In addition, the nurse midwives who carried out the experimental intervention knew that the participants were studied, but were unaware of the process, the variables and the degree of antenatal risk in the women.

\section{Sample and data}

The study used a sample of pregnant women, with low and precarious socioeconomic levels considered at postpartum depression risk (using the interview validated by Righetti-Veltema et al. [31]. A total of $n=529$ women were recruited using the computerized scheduling of echography. After the test, information about the study 
was provided and a formal consent was given. Only participants with moderate or high risk $(n=220)$ were assigned by computer program (SPSS.15), to a control group (CG) or an experimental group (EG), resulting in nine intervention groups and their respective control groups. For the random allocation sequence simple random sampling was used.

The selection criteria were: a) less than 20 weeks pregnant, b) moderate or high risk of depression, c) primiparous or multiparous with fewer than three children, d) have a partner, e) have lower socioeconomic status (classification Feito 1995) and f) language comprehension. We excluded women without psychosocial risk, without partner, those who consumed drugs, who diagnosed from mental disorders, who belonged to a high social class, and not understand the language.

The study was conducted in three health centers located in three different European cities. The antenatal groups took place in the period 2003-2007; the data were analyzed during 2008-2010 by additional analysis in 2012. The three study cities (Barcelone, Figueres, Beziers) have a high percentage of immigrants; the birth rate, the living conditions and access to health services are proportionate. In the centers studied, the standard maternal education program is comparable with the same theoretical foundation. In addition, these centers have a wide range of clients who live in urban areas or in the suburbs; therefore, the probability of any coincidental or prior relationship between the participants was low.

Antenatal EG groups started when women were between weeks 20-28 of pregnancy. The EG women received weekly sessions provided by a phone call between sessions for recording incidents and strengthening participation. CG women attended the usual antenatal sessions, or the standard eight sessions, from the $30^{\text {th }}$ week of pregnancy; they received no phone call between the sessions. Some women did not participate in any program; they were free to leave the program participation in all cases. All participants received a layette for compensation together with the postnatal questionnaires by parcel post.

\section{Intervention}

Tourne's model showed that nursing intervention in antenatal experimental groups is based on Roger's humanistic values [32]; according to Tourne's model, the learning for the women and men who are expecting a baby is very intense, because it covers a personal process for each, there is also a change of family and of roles: couple, social life... all in just nine months. For some parents there is added economic hardship or cultural change, with rapid adaptation full of events. The Tourne psychosomatic approach is based in the idea that the body and mind are a unity indisociable. If the pregnant women suffer from excessive somatic complaints it is highly likely to be affected by psycho emotional experiences or problems. A similar idea is Kamysheva et al. study, in which a higher frequency of physical symptoms was associated with increased depressive symptoms in pregnant women [29].

The nursing-midwifery process is focused on goals that require actions to achieve a state of good health; for this the nurse-patient relationship is the essential framework. The beliefs, values, attitudes and knowledge of the patient and of the practitioner serve to explore and understand the dynamics of health. Ultimately, each person sees himself/herself as having the resources and capabilities, putting aside the confusing advice of other lay people, and having the means of a very attentive professional accompaniment. The most unique feature of the program interventions under study is the use of a conscious model tailored to the individual needs and circumstances of the participants; including the consciousness and differentiation of feelings/sensations, the ability to resolve symptoms by understanding themselves ultimately results in greater autonomy and responsibility; in this approach each person finds their own answers. The theoretical basis of the method also includes a cognitive model that results in the exchange of ideas and beliefs about pregnancy and childbirth among participants; it also retains the attachment theory to allusions from that point out the existence of the baby in utero from the first session. In short, the approach is friendly, comprehensive and integrative.

Three nurses-midwives previously schooled by the study investigators, not familiar the sampling process or the questionnaires, conducted the experimental program sessions. Nurses-midwives received 20 hours of training. A notebook with a scheme of the content, objectives and process to follow in each session served as a guide. The sessions were taped with the consent of the participants, the purpose was to evaluate the content of the intervention and ensure the same application in all groups.

In control group, women receive the program standard centered on the control of pain in childbirth and recommendations for care the child. There is no taped in sessions. The sessions times and the number of sessions appliqued is similar.

\section{Outcomes measure}

The selection interview used came from the authors RighettiVeltema et al. [31] and contains psychosocial variables: the origin, economic status, relationship with one's mother, perception of the pregnancy (positive or negative), willingness to prepare oneself for the birth, somatic symptoms, and psychological treatment history. Each question has a value at the end ranging from zero to nine points; risk is considered from three points onward.

The battery of questionnaires consisted of: a) EPDS scale [33], validated Spanish version [34], the cutoff point of the Edinburg postpartum depression symptoms used to identify the risk of PPD was $\geq 12$; b) level of social support (Functional Social Support Questionnaire survey of Broadhead et al, 1988) [35], validated Spanish version [36], c) stress events (Holmes and Rahe, 1967) [37], adapted version [38] and d) The relationship of couples (DASS Spanier, 1976) translated into Spanish and validated [39]. In the second evaluation, after delivery $\left(\mathrm{T}_{2}\right)$ was used in addition, a questionnaire on follow-up care and birth outcomes (health of mother and baby including birth weight and gestational week at birth, type of delivery and problems of the same).

\section{Statistical analysis}

For data analysis the criteria intention of simple treatment are considered. Analyses were performed by integrating the control group and the experimental group evaluating depressive symptoms, social support, stress, partner relationship and postnatal outcomes. For the analysis of differences between groups the Pearson X2 test, the U de Mann-Whitney test or the Kolmogorov-Smirnov test was used, the normality of the variables was analyzed using the KolmogorovSmirnov test. Statistical significance was set at $\mathrm{P}<0.05$ and the confidence interval was set at $95 \%$. Quantitative variables were expressed as mean with standard deviation (SD) and dichotomous as a percentage. For quantitative variables the student test for comparison 
of means was used for independent samples (intergroup differences) and paired samples (intragroup differences). We used the software package SPSS.15 always.

\section{Results}

A total of $\mathrm{N}=529$ interviews were conducted from which we selected $\mathrm{n}=220$ cases of risk, rejecting the cases with low and without risk. Were excluded $n=36$ women, seven by moving to another country, four by abortion and two for medical reasons-these were justified, there were 23 unjustified dropouts, including four with apparent violence, nine lost cases and ten cases that would not continue. The study included n=184 women. Finally, of the 147 questionnaires received postpartum only 127 cases $(69.02 \%)$ were analyzed for response errors. $31 \%$ of the sample was lost (Figure 1).

\section{Maternal characteristics}

The demographic data of the participants were comparable in both groups, showing no significant difference between the centers studied. The mean age was $\bar{x}=29.3$ years ( 28.36 to 30.25 ) to $5.53 \mathrm{SD}$; there were 11 women between 18-20 years, a woman of 42 years and another of 43 years. Regarding the origin, 50.39\% (64) of the participants were Spanish, $4.72 \%$ (6) were from another European country and $44.89 \%$ (57) came from another continent; by adding the non-Spanish Europeans with those from other continents, we obtained a sample of half Spanish and half foreign nationals. The education profile was $14 \%$ (18) with primary education, 29\% (38) with secondary education, $14.9 \%$ (19) with some period of training, $16.40 \%$ (21) with full professional training, $14 \%$ (18) said they had access to college, and finally, $11.7 \%$ (12) had completed college. Socioeconomic status was $14.13 \%$ (18) middle class, $24.73 \%$ (31) situation between medium and low, $34.86 \%$ (45) working class and $26.28 \%$ (33) below the threshold poverty. Parity was $65.41 \%$ (75) primiparous and $34.58 \%$ (52) multiparous. The variable pregnancies indicated that $22.3 \%$ (32) of the participants had a history of abortion, with an average of previous pregnancies $\bar{x}=2(1.3 \mathrm{SD})$.

\section{Satisfaction with the models}

All participants were very similar good satisfied with the antenatal program in both groups when asking about satisfaction with the preparation for childbirth overall. We exposed the affirmative responses of participants ("yes, it helped me"; "yes, I'm satisfied") in present work; they was completed the questionnaire together with partner as received set point try to answer in consensus.

The percentage of fully completed satisfaction questionnaire from CG participants was $15 \%$ (only $25 \%$ participated more than seven sessions in a standard intervention) and from the EG was $85.70 \%(88 \%$ participated more than seven of the ten sessions); these comparative analyses were evaluated with the chi square test and the Fisher test. The results obtained are as follows:

- There is a significant difference between the groups for questions about understand, expressing feelings and communicating with baby in uterus: understanding the symptoms of pregnancy: 4 in the CG and 38 in the EG ( $\mathrm{p}=0.05$ Pearson Chi-Square Test and $\mathrm{p}=0.05$ Fisher's Test); understanding all information received: 3 in the CG and 37 in the EG ( $\mathrm{p}=0.02$ Pearson Chi-Square Test and $\mathrm{p}=0.02$ Fisher's Test); expressing my feelings: 0 in the CG and 18 in the EG ( $p=0.04$ Pearson Chi Square Test and $p=0.03$ Fisher's
Test); and, communicating with the baby: 3 in the CG and 38 in the EG ( $\mathrm{p}=0.05$ in Pearson Chi-Square Test and $\mathrm{p}=0.05$ Fisher's Test).

- No difference is noted for questions relative at cognitive objectives, loneliness, social support and communication: clarifying information about pregnancy ( 4 in the CG and 27 in the EG: $\mathrm{p}=0.45$ ); changing erroneous beliefs ( 4 in the CG and 31 in the EG: $\mathrm{p}=0.35$ ); reducing my loneliness ( 4 in the $C G$ and 30 in the EG: $\mathrm{p}=0.37$ ); couple communication ( 3 in the CG and 21 in the EG: $\mathrm{p}=0.70$ ); improving support in the couple ( 1 in the CG and 21 in the EG: $p=0.10$ ); requesting overall support ( 0 in the $C G$ and 9 in the EG: $\mathrm{p}=0.16$ ).

\section{Outcome of variables: social support, stress, and relationship with partner}

The variables social support and stressful events do not indicate any change between antenatal and postnatal evaluation in either group, no difference was found $(\mathrm{p}=0.26$ and $\mathrm{p}=0.58$ respectively in the CG, $\mathrm{p}=0.92$ and $\mathrm{p}=0.19$ respectively in the $\mathrm{EG}$ ). No differences were observed between groups in any of the variables assessed in the postpartum.

\section{Premature births and weight of the newborns}

In total there were $\mathrm{n}=16$ preterm births, $13(22.4 \%)$ in the CG and three $(4.4 \%)$ in the $E G$, the difference is significant $(\mathrm{P}=0.003)$ when the $\mathrm{p}$-value is calculated with the Pearson X2 test, there is no independence between the two variables, the group is important. Among the premature infants, six were born between 29-33 weeks, five in the CG and only one in the EG (due to premature rupture of membranes). The analysis by weeks of gestation to birth of the baby shows that there are differences in the level of prematurity of the infants studied. The p-value comparison test between the means of the groups indicates a significant difference $(\mathrm{p}=0.02)$ when calculated with the Student $\mathrm{t}$ test. The mean of the variable weeks of gestation is lower in the CG with greater deviation: $\bar{X}=39.20$ (2.88 SD) comparatively to the EG: $\bar{X}=40.40$ (1.50 SD). However, to implement the contrast in nonparametric version with test Mann-Whitney $U$, the $p$-value of $\mathrm{p}=0.08$. Probably the size of the sample influences these analyses. In relation to the weight of the baby at birth, an analysis the average statistics suggests that the CG babies weighed less than the others (almost 300g). The average weight of the babies was $\bar{X}=3019.01$ (668.83 SD) for the GC and for the babies of the EG it was: $\bar{X}=3301.87$ (506.65 SD). The average weight of the EG is higher while the standard deviation is less than that of the CG. The p-value of the parametric comparison test calculated with Student's $t$ is $p=0.01$, leading to the conclusion that the average variable weight of the GE is significantly higher than that of GC.

\section{Results from adherence to the program and adherence to the antenatal care protocol}

The adherence to the experimental program was $88 \%$ (60) participated $\geq 7$ sessions, and $46 \%$ (32) of the participants attended all sessions. Those who participated more than four sessions showed a clear reduction of postnatal depressive symptoms compared to the antenatal evaluation in a univariate analysis, this difference was significant in the analysis with the ' $\mathrm{t}$ ' of Student $(\mathrm{p}=0.002)$ and also significant in the analysis with the Wilcoxon test $(\mathrm{p}=0.003)$. That is, women who participated in at least half of the sessions had a 
significantly lower mean EPDS than in the antenatal assessment. There was no greater impact to more participation; a similar effect was obtained from five sessions. Curiously women from other countries participated more than the local women.

Adherence to the protocol was assessed from the visits, usually identical to the protocol of each of the study centers. In $64.7 \%$ of the cases, the women of the CG made all protocol visits, compared to $79.6 \%$ of the participants of the EG, although there is greater adherence than the EG, the difference did not reach statistical significance $(\mathrm{p}=0.080)$. In relation to the execution of the analyses and tests such as ultrasound, $84.1 \%$ of CG women attended in comparison to $92 \%$ of participants in the EG, with no significant difference $(\mathrm{p}=0.211)$. The most remarkable and significant variable is the assistance to men in the antenatal preparation sessions at birth, $23.1 \%$ of the CG men participated in three sessions compared to over $60 \%$ of men participation in the EG $(\mathrm{p}<0.0001)$.

\section{Discussion}

In the evaluated antenatal program, significant data were obtained concerning the babies' weight and the premature birth, as well as adherence to the program, an indicator of the women's satisfaction. This adherence to the experimental program could explain that women made more antenatal care, more health checks and, in turn, they could affect the baby's weight. However, caution should be taken in the conclusions due to the loss of questionnaires, sometimes questionnaires were not returned, and at other times responded to mistakenly; the self-assessment was risky. An interviewer facilitated the execution of responses and avoided the loss of questionnaires; unfortunately there was no funding for it. This loss did not help to achieve statistical significance.

In analyzing the profile of the missing cases, two variables were highlighted: the origin and socioeconomic status, both are significantly different between the initial participants and those lost $(\mathrm{p}=0.001)$, curiously, more were lost of the Spanish and the more disadvantaged socioeconomic status participants $(\mathrm{p}<0.001)$. Age indicates a difference in the limit of significance $(p=0.05)$. Neither parity nor gestation shows significant differences between subjects who remained in the study and those who were lost (parity: $\mathrm{p}=0.436$; gestation: $\mathrm{p}=0.352$ ). There were no significant differences in the level of education between the women who left and those who remained in the study $(\mathrm{p}=0.07)$. Some of these data contradicted those observed in a study of about the participation in antenatal programs whereby, age and origin are variables that make a difference, while the oldest and the Spanish were those who participated more [6], however, the same study coincides with ours with the smaller participation of the poorest women. Importantly, this study was conducted in areas with high immigration rates (15-30\%), a conscious choice to ensure the participation of people of the lower social class. The rate of immigrants certainly influences the results, and they relocated frequently. It happened that foreigners benefited more than those of the country, they were even more involved than expected. The study helped establish links between mothers. Although the precariousness of economic funding slowed and hindered the process of the study, the benefits on people and the satisfaction described by participants was extraordinary.

Losses in randomized studies with pregnant women are common, the loss rate can reach $41 \%$, regardless of the type of intervention, especially if there is no compensation; the young, those with less education, those with lower incomes, with less social support, smokers, and those with a history of depression are the most abandoned according to an exhaustive Canadian study [40].

Pregnant women in living in difficult circumstances in the social and economic level and lacking a healthy environment, may remain outside antenatal groups due to their own discouragement and isolation; care for these women is needed to care for their infants [41]. An antenatal group is a way to encourage support for different levels and if one can help curb some of prematurity, then it would be a good option in cases at risk. However, the program may have to include more than ten sessions, additional individual interventions in certain women, extension of the approach after birth, or interfere with an interdisciplinary team as proposed by Halbreich [42]

The association between socioeconomic status, stressful events and depression before and after childbirth has been well explored [43], in addition, the loss of social support resulting from death of loved ones or marital conflict, low material support due to economic hardship or living in poverty favors stress [44], a situation that affected the participants in present study. In any case, we find that the decrease in preterm deliveries and the adherence is high, so that, somehow, the results are encouraging.

\section{Conclusions}

The antenatal program, evaluated by applying a Tourné intervention and a protocolled content, opens avenues of study that have not yet been explored. The obtained results suggest that the prematurity in women of low socioeconomic status needs to be studied with a much larger sample exposure, adding incentives to encourage retention in the study for the control cases. Early detection performed exploiting women's visits to the echography before the $22^{\text {nd }}$ week was a bonus and suggests effectiveness in the selection of cases at risk, because in our country, women of all conditions perform that test. Prematurity, the weight of the babies and adherence to the program can permit to conclude that the study conducted had an effect. It would be appropriate to continue investigating in this line with a larger sample and avoiding the use of mail for data collection; mailed self-administered questionnaires constitute a limit, because in addition to the risk of loss, they can be answered by other people. Finally, the results of this study must be understood within the limits explained. The overall contribution of the research is that much less interesting and novel, and suggests a different approach to the preparation of care at childbirth for the benefit of the variables addressed. The antenatal sessions that provide information and birth preparation exercises are appropriate in the general population, but women at psychosocial risk should be offered different approaches. The association between pregnancy processes and results thereof (premature birth, low birth weight baby) involves a multidimensional approach needed to address the care of the mother and the baby.

\section{Acknowledgements}

Our deepest gratitude goes to all the participants and their partners who showed great sympathy and respect for the study at any time. An investigation of this magnitude is only possible if the leaders most responsible for clinical and health services work together, in this case the availability shown by all in the three centers must be recognized. Finally, the most deserved recognition goes to the nurse midwives ( $M$. Arbucies, A. Sanés, A. Juanola and A. Ferrini) who ran the program 
and thanks to the ultrasound service secretaries who made the development of the fieldwork a success.

\section{Additional information}

A) The social classification used in this study corresponds to: Feito $\mathrm{R}$ (1995) Contemporary social structure. Social classes in industrialized countries. Siglo XXI Editores SA, Madrid.

B) The original reference questionnaire on the relationship is: Spanier GB (1976) Measuring Dyadic Adjustment: New Scales for Assessing the Quality of Marriage and Similar Dyads. J Marriage and Family 38: 15-28.

\section{References}

1. Sanchez Y, Muñoz N, Pérez S, Robledo A, Pallás CR, et al. (2003) Mujeres inmigrantes. Immigrant women: characteristics of antenatal care and childbirth. Prog Obstet Gynecol 46: 441-447.

2. Chung EK Mathew L, Elo IT, Coyne JC, Culhane JF (2008) Depressive symptoms in disadvantaged women receiving prenatal care: the influence of adverse and positive childhood experiences. Ambul Pediatr 8: 109-116.

3. de la Torre J Coll C, Coloma M, Martín JI, Padrón E, et al. (2006) [Antenatal care in immigrants]. An Sist Sanit Navar 29 Suppl 1: 49-61.

4. Yelland J Sutherland G, Brown SJ (2010) Postpartum anxiety, depression and social health: findings from a population-based survey of Australian women. BMC Public Health 10: 771.

5. Mustard CA Roos NP (1994) The relationship of prenatal care and pregnancy complications to birthweight in Winnipeg, Canada. Am Public Health 84: 1450-1457.

6. Paz-Pascual C Pinedo IA, Grandes G, de Gamboa GR, Hermosilla IO, et al. (2008) Design and process of the EMA Cohort Study: the value of antenatal education in childbirth and breastfeeding. BMC Nurs 7: 5 .

7. Halle C Dowd T, Fowler C, Rissel K, Hennessy K, et al. (2008) Supporting fathers in the transition to parenthood. Contemp Nurse 31 : 57-70.

8. Blatt AJ Lieberman JM, Hoover DR, Kaufman HW (2012) Chlamydial and gonococcal testing during pregnancy in the United States. Am Obstet Gynecol 207: 55.

9. Diego MA, Field T, Hernandez-Reif M (2005) Pre-partum, postpartum and chronic depression effects on neonatal behaviour. Infant Behav Dev 28: 155-64.

10. Field T Diego M, Hernandez-Reif M (2006) Prenatal depression effects on the fetus and newborn: a review. Infant Behav Dev 29: 445-455.

11. Marcus SM (2009) Depression during pregnancy: rates, risks and consequences--Motherisk Update 2008. Can J Clin Pharmacol 16: e15-22.

12. Matthey S Kavanagh DJ, Howie P, Barnett B, Charles M (2004) Prevention of postnatal distress or depression: an evaluation of an intervention at preparation for parenthood classes. J Affect Disord 79: 113-126.

13. Vieten C Astin J (2008) Effects of a mindfulness-based intervention during pregnancy on prenatal stress and mood: results of a pilot study. Arch Womens Ment Health 11: 67-74.

14. Zlotnick C Miller IW, Pearlstein T, Howard M, Sweeney P (2006) A preventive intervention for pregnant women on public assistance at risk for postpartum depression. Am J Psychiatry 163: 1443-1445.

15. Lara MA Navarro C, Navarrete L (2010) Outcome results of a psychoeducational intervention in pregnancy to prevent PPD: a randomized control trial. J Affect Disord 122: 109-117.

16. Field T Diego M, Hernandez-Reif M, Deeds O, Figueiredo B (2009) Pregnancy massage reduces prematurity, low birthweight and postpartum depression. Infant Behav Dev 32: 454-460.

17. Facchinetti F, Ottolini F (2004) Stress and preterm delivery. J Psychosom Obstet Gynaecol 25: 1-2.
18. Behrman RE, Butler AS (2006) Preterm Birth: Causes, Consequences, and Prevention. Institute of Medicine Committee on Understanding Premature Birth and Assuring Healthy Outcomes. The National Academies Press Washington, DC

19. Saisto T Salmela-Aro K, Nurmi JE, Könönen T, Halmesmäki E (2001) A randomized controlled trial of intervention in fear of childbirth. Obstet Gynecol 98: 820-826.

20. Muñoz RF, Le HN, Ghosh IC, Diaz MA, Urizar GG, et al. (2007) Prevention of Postpartum Depression in Low-Income Women: Development of the moms and babies / Mothers and Babies Course. Cogn Behav Pract 14: 70-83.

21. Austin MP, Frilingos M, Lumley J, Hadzi-Pavlovic D, Roncolato W, et al. (2008) Brief antenatal cognitive Behaviour therapy group intervention for the prevention of postnatal depression and anxiety: A randomized controlled trial. J Affec Disord 105: 35-44.

22. Le HN Perry DF, Stuart EA (2011) Randomized controlled trial of a preventive intervention for perinatal depression in high-risk Latinas. J Consult Clin Psychol 79: 135-141.

23. Spinelli MG Endicott J (2003) Controlled clinical trial of interpersonal psychotherapy versus parenting education program for depressed pregnant women. Am J Psychiatry 160: 555-562.

24. Grote NK, Swartz HA, Bledsoe SE, Frank E (2004) Treating depression in low-income pregnant patients: The role of brief interpersonal psychotherapy. Social Work Prac Res 14: 397-406.

25. Buist A, Westley D, Hill C (1999) Antenatal prevention of postnatal depression. Women Health, 1: 167-173.

26. Brugha TS Wheatley S, Taub NA, Culverwell A, Friedman T, et al. (2000) Pragmatic randomized trial of antenatal intervention to prevent postnatal depression by reducing psychosocial risk factors. Psychol Med 30: 1273-1281.

27. Hayes BA Muller R (2004) Prenatal depression: a randomized controlled trial in the emotional health of primiparous women. Res Theory Nurs Pract 18: 165-183.

28. Tourné CE (2002) Obstétrique psychosomatique et fonctionnelle. Medical Sauramps. Montpellier, France.

29. Kamysheva E Skouteris H, Wertheim EH, Paxton SJ, Milgrom J (2008) Examination of a multi-factorial model of body-related experiences during pregnancy: the relationships among physical symptoms, sleep quality, depression, self-esteem, and negative body attitudes. Body Image 5: 152-163.

30. Rauff EL Downs DS (2011) Mediating effects of body image satisfaction on exercise behavior, depressive symptoms, and gestational weight gain in pregnancy. Ann Behav Med 42: 381-390.

31. Righetti-Veltema M, Conne Perréard E, Bousquet A, Manzano J (2006). Construction et validation d'un questionnaire multicentrique pour le dépistage de la dépression anténatale et postnatale. Enfant Psychiat. 49: 513-541.

32. Rogers C, Kinget M (1971) Psicoterapia y relaciones humanas. Alfaguara: Madrid, Espagne.

33. Cox JL, Holden JM, Sagovsky R (1987) Detection of postnatal depression: Development of the 10-item Edinburgh Postnatal Depression Scale. Br J Psychiatry 150: 782-786.

34. Ascaso Terrén C, Garcia Esteve L, Navarro P, Aguado J, Ojuel J, Tarragona MJ (2003). Postpartum Depression Prevalence in a Spanish sample: comparation of estimations with Structured Clinical Interview for DSM-IV against the Edinburgh Postnatal Depression Scale (EPDS) Clin Med 120: 326-329.

35. Broadhead WE, Gehlbach SH, De Gruy FW (1988) The Duke-UNC Functional Social Support Questionnaire. Measurement of social support in Family Medicine patients. Med Care 1988; 26: 709.

36. Bellón Saameño JA Delgado Sánchez A, Luna del Castillo JD, Lardelli Claret P (1996) [Validity and reliability of the Duke-UNC-11 questionnaire of functional social support]. Aten Primaria 18: 153-156, 158-63. 
Citation: Assumpta OCM (2014) Comparing a Novel Antenatal Program with a Standard in Disadvantaged Women at Psychosocial Risk: A Randomized Controlled Study. Clinics Mother Child Health 11: 165. doi:10.4172/2090-7214.1000165

Page 8 of 8

37. Holmes TH, Rahe RH (1967) The social readjustment rating scale. Journal of Psychosomatic Research 11: 213-218.

38. Gonzalez de Rivera JL, Morera A (1983) Assessment of life events: Spanish adaptation of the Holmes and Rahe scale. Psyche 7: 20-25.

39. Pérez S (2001) Psychotherapy partner. Longitudinal study [PhD thesis]. Barcelona: Ramon Llull University of Barcelona.

40. Tough SC Siever JE, Johnston DW (2007) Retaining women in a prenatal care randomized controlled trial in Canada: implications for program planning. BMC Public Health 7: 148.

41. Nzama B Hofmeyr J, Smith H, Garner P, Brown H, et al. (2005) Improving the experience of birth in poor communities. BJOG 112: 1165-1167.
42. Halbreich U (2005) The association between pregnancy processes, preterm delivery, low birth weight, and postpartum depressions--the need for interdisciplinary integration. Am J Obstet Gynecol 193: 1312-1322.

43. Alami KM Kadri N, Berrada S (2006) Prevalence and psychosocial correlates of depressed mood during pregnancy and after childbirth in a Moroccan sample. Arch Womens Ment Health 9: 343-346.

44. Pereira PK, Lovisi GM, Pilowsky DL, Lima LA, Legay LF y L (2009) Depression during pregnancy: Prevalence and risk factors among women attending a public health clinic in Rio de Janeiro, Brazil. Cad Saude Publica 25: 2725-2736. 